\title{
Escala diagramática para avaliação de mofo cinzento (Amphobotrys ricini) da mamoneira (Ricinus communis L.)
}

\author{
Haroldo Antunes Chagas ${ }^{2 *}$; Marco Antonio Basseto ${ }^{1}$; Daniel Dias Rosa ${ }^{1}$; Maurício Dutra Zanotto ${ }^{2}$; Edson Luiz Furtado ${ }^{1}$
}

${ }^{1}$ Departamento de Produção Vegetal/Defesa Fitossanitária e 2Departamento de Produção Vegetal/Agricultura e Melhoramento Vegetal, Universidade Estadual Paulista - UNESP, Faculdade de Ciências Agronômicas - FCA - Rua José Barbosa de Barros, 1780, Cx. Postal 237, CEP 18610-307, Campus Botucatu, Botucatu, SP, Brasil.

Autor para correspondência: Haroldo Antunes Chagas (haroldo.antunes@yahoo.com.br)

Data de chegada: 31/08/2009. Aceito para publicação em: 10/05/2010.

\section{RESUMO}

Chagas, H.A.; Basseto, M.A.; Rosa D.D.; Zanotto, M.D.; Furtado, E.L..Escala diagramática para avaliação de mofo cinzento (Amphobotrys ricini) da mamoneira (Ricinus communis L.) Summa Phytopathologica, v.36, n.2, p.164-167, 2010.

O objetivo do trabalho foi a elaboração de uma escala diagramática para avaliação de mofo cinzento causado por Amphobotrys ricini (Buchw.) em mamoneira (Ricinus communis L.). Utilizaram 59 cachos, que foram desinfestados em solução de hipoclorito de sódio a $2 \%$ por 30 segundos e em água destilada e esterilizada. Depois foram acondicionados em bandejas com espuma umedecida, onde receberam discos de micélio de $5 \mathrm{~mm}$ do patógeno, permanecendo em câmara climática a $25^{\circ} \mathrm{C}$ e UR de $80 \%$. Observou- se a evolução da doença e foram obtidos fotos dos cachos doentes diariamente. Para a determinação da porcentagem de severidade dos cachos, os frutos infectados e sadios foram contados, estimando-se dessa forma a porcentagem da área lesionada e elaborando uma escala diagramática com seis níveis de severidade. A adoção da escala proposta, melhorou a acurácia $\left(R^{2}=0,94\right)$, com valores de "a" não significativamente diferentes de zero $(0)$ e os valores de "b" não diferentes de um (1).

Palavras-chave adicionais: avaliação, severidade, acurácia, precisão.

\section{ABSTRACT}

Chagas, H.A.; Basseto, M.A.; Rosa D.D.; Zanotto, M.D.; Furtado, E.L.. Diagramatic scale to assess gray mold (Amphobotyrs ricini) in castor bean (Ricinus communis L.). Summa Phytopathologica, v.36, n.2, p.164-167, 2010.

The aim of the present study was to develop a diagrammatic scale to assess gray mold caused by Amphobotrys ricini in castor bean (Ricinus communis L.). The experiment included 59 clusters, which were disinfected in solution of sodium hypochlorite to $2 \%$ for 30 seconds and distilled water and sterilized. Then, they were packed in moistened foam trays which received $5 \mathrm{~mm}$ mycelial discs, and were kept in a climatic chamber at $25^{\circ} \mathrm{C}$ and $80 \%$. RH. The disease

Keywords: evaluation, severity, accuracy, precision

A mamoneira (Ricinus communis L.) pertence a família Euphorbiaceae e gênero monotípico, ou seja, é a única espécie do gênero Ricinus. É uma espécie preferencialmente autógama, mas pode apresentar alta taxa de cruzamentos, podendo chegar a $40 \%$ de acordo com Beltrão et al. (3).

Dos produtos obtidos, o óleo é o mais explorado comercialmente. Devido à alta capacidade de reações químicas dadas pelo ácido graxo ricinoléico, o óleo tem larga aplicação na fabricação de tintas, vernizes, detergentes, inseticidas, resinas de plástico, lubrificantes, fungicidas e bactericidas, próteses humanas para coluna vertebral, crânio, dentes e mamas $(6,8)$.

Embora seja uma planta com grande capacidade de adaptação às mais diferentes regiões do mundo, a mamoneira está sujeita a doenças causadas por diversos microorganismos, os quais causam grandes prejuízos econômicos $(7,17)$.

Entre essas doenças, pode-se destacar o mofo-cinzento, causado evolution was observed daily pictures of the clusters diseased. To determine the severity percentage of the bunches, infected and healthy fruits were counted, estimating thus the percentage of the injured area, and developing a diagrammatic scale with six severity levels. The adoption of the proposed scale, improved the accuracy $\left(\mathrm{R}^{2}=0,94\right)$ "a" values were not significantly different from zero $(0)$ and "b" values were not significantly different from one (1). pelo fungo Amphobotrys ricini (Buchw.) Hennebert (sin. Botrytis ricini N.F. Buchw.) (Teleomórfico Botryotinia ricini (G.H. Godfrey) Whetzel, sendo a principal doença da cultura da mamoneira. O patógeno encontra-se disseminado em todas as áreas de cultivo dentro do território brasileiro, ocorrendo agressivamente sempre que as condições climáticas são favoráveis. Essas condições são temperatura ao redor de $25^{\circ} \mathrm{C}$ e Umidade Relativa do ar acima de $75 \%$. A doença causa grandes prejuízos à produção, afetando inflorescências, cachos e frutos, reduzindo assim a produção de óleo pela diminuição dos frutos colhidos e ,sua qualidade segundo Lima et al. (13).

A principal forma de disseminação do patógeno é por esporos carreados pelo vento e por sementes, de acordo com Neergaard (15). Porém alguns insetos, atraídos pela grande exsudação de néctar nas flores, também desempenham papel na disseminação de A. ricini (11, 14).

Os primeiros sintomas são pequenas manchas de tonalidade 
azulada nas inflorescências e nos frutos em desenvolvimento. Em um segundo estágio e em condições climáticas favoráveis, o patógeno inicia o ciclo reprodutivo, podendo-se observar sua esporulação na superfície dos tecidos afetados, conferindo assim à área lesionada um aspecto pulverulento de coloração cinza-escura, segundo Godfrey (9).

Atualmente, não existe na literatura uma forma adequada para se avaliar a severidade desta doença em condições de campo. A elaboração de uma escala diagramática apresenta uma série de dificuldades, devido ao principal órgão da planta afetado, o fruto, ser de difícil avaliação, mas uma escala é uma importante ferramenta para se quantificar doença e assim verificar seu impacto sobre a cultura.

Campbell \& Madden (5) descreveram dois termos freqüentemente utilizados na quantificação da severidade de doenças que são acurácia e precisão. Acurácia refere-se à proximidade do valor estimado, neste caso o valor prédito na escala diagramática, com o valor real de quantidade de doença avaliada no órgão, enquanto precisão refere-se à variação ou repetibilidade associadas com uma estimativa.

De acordo com Bergamim \& Amorin (4), a quantificação da severidade está sujeita a grande subjetividade, pois normalmente é estimada visualmente e pode variar muito entre os avaliadores. A utilização de escalas diagramáticas reduz a subjetividade das estimativas de severidade entre os avaliadores, melhorando os níveis de acurácia e precisão. As escalas diagramáticas são ainda de fácil uso, aplicáveis em diferentes condições e geram resultados reproduzíveis, além de apresentarem, de forma clara, todos os estádios de desenvolvimento da doença.

Considerando a inexistência de métodos padronizados para quantificação do mofo-cinzento da mamoneira, este trabalho teve como objetivo desenvolver uma escala diagramática para a avaliação da severidade da doença e efetuar sua validação em campo, a fim de melhorar os valores de acurácia e precisão da doença com sua utilização.

\section{MATERIAL E MÉTODOS}

\section{Elaboração de escala diagramática para avaliação de mofo cinzento}

Para a elaboração da escala diagramática do mofo cinzento foram coletados 59 cachos de mamoneira (cv. Al-Guarany) em uma área da Fazenda Experimental Lageado da FCA - UNESP, no município de Botucatu-SP, sendo esses encaminhados para o Departamento de
Produção Vegetal, setor de Defesa Fitossanitária. Os cachos foram desinfestados com uma solução de álcool $70 \%$, por 30 segundos, solução de hipoclorito de sódio a $2 \%$, por 30 segundos e lavados em água destilada esterelizada. Em seguida, os mesmos foram distribuídos em bandejas plásticas, contemplando quatro cachos por bandeja. A seguir, discos de $5 \mathrm{~mm}$, obtidos de uma cultura pura do micélio de $A$. ricini, cultivado em meio BDA (Batata - Dextrose - Agar), a $25^{\circ} \mathrm{C}$, por 8 dias, foram alocados na superfície dos cachos e a bandeja foi acondicionada em uma câmara com a umidade relativa do ar maior que $80 \%$ e temperatura de $25^{\circ} \mathrm{C}$. Observou-se a evolução da doença, através de fotos tiradas diariamente dos cachos. Para a determinação da porcentagem de severidade dos cachos (severidade real), os frutos infectados e sadios foram contados, estimando a porcentagem da área lesionada e elaborando uma escala diagramática com seis níveis de severidade.

É importante ressaltar que a escala diagramática seguiu a "Lei de Weber-Fechner" (incrementos logarítmicos), sem necessariamente utilizar intervalos escolhidos por Horsfall \& Barratt (10). Assim, os níveis propostos permitem uma melhor diferenciação visual da severidade da doença no cacho da mamoneira.

\section{Validação da escala diagramática}

No teste de validação da escala diagramática foram utilizadas 93 fotos dos cachos com sintomas de mofo-cinzento com diferentes níveis de intensidade da doença. A severidade foi estimada por quatro avaliadores, sem e com o auxílio da escala diagramática, sendo, posteriormente comparada a severidade real previamente obtida.

Tanto a acurácia, quanto a precisão de cada avaliador, foi determinada por meio de regressão linear simples, com a severidade real obtida eletronicamente como variável independente e a severidade estimada pelo avaliador como variável dependente. A acurácia das estimativas foi determinada pelo teste $\mathrm{T}$ aplicada ao intercepto da regressão linear (a), para verificar se foi significativamente diferente de 0 , e ao coeficiente angular da reta (b), para testar se foi significativamente diferente de 1 , ao nível $1 \%$ de probabilidade. Valores de intercepto significativamente diferentes de 0 indicam a presença de desvios sistemáticos. A precisão das estimativas foi obtida pelo coeficiente de determinação da regressão $\left(\mathrm{R}^{2}\right)$ e pela variância dos erros absolutos (severidade estimada menos severidade real). As análises de regressão foram efetuadas com o auxílio do programa Microsof Excel 2000 ${ }^{\circledR}$ (Microsoft Corporation, 2000).
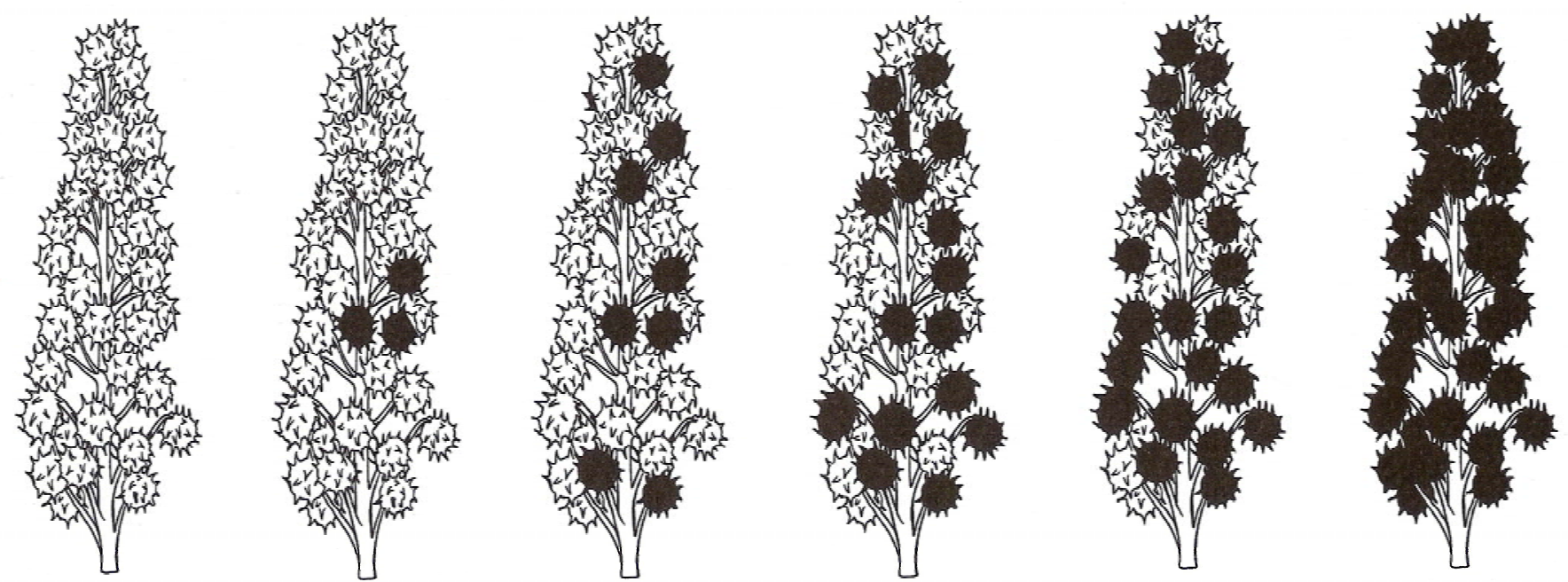

Figura 1. Escala diagramática desenvolvida para avaliação de mofo cinzento causado por Amphobotrys ricini em cachos de mamoneira indicando os níveis de $0 ; 8 ; 22 ; 43 ; 76$ e $100 \%$ de severidade (porcentagem de cacho infectado). 
Sem escala
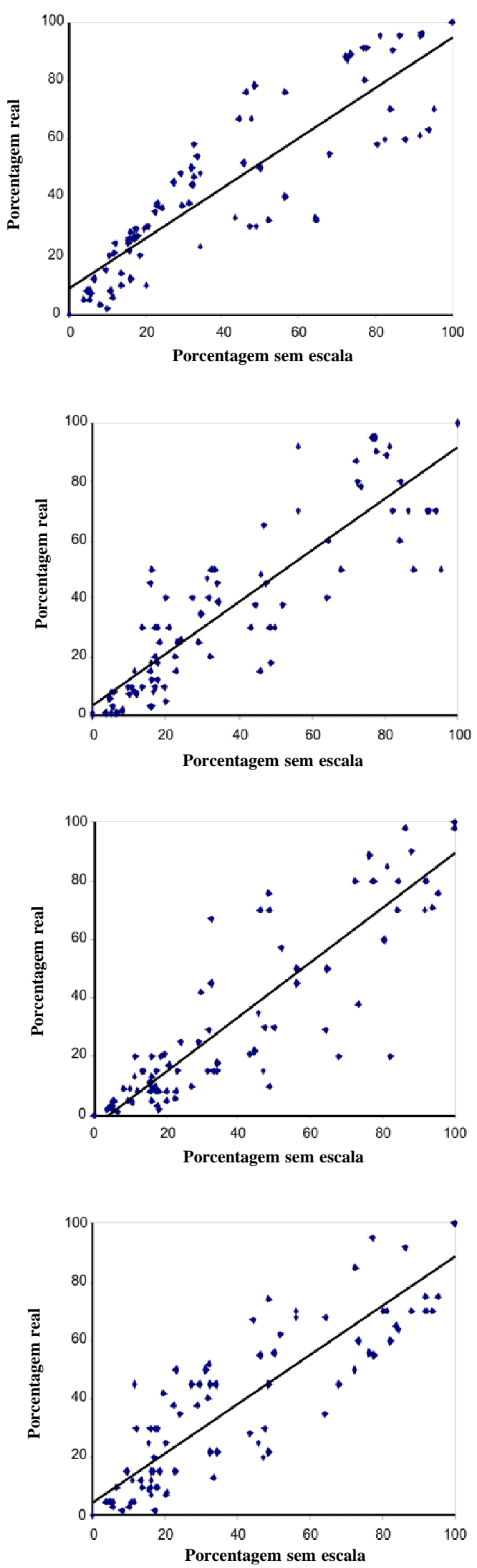

$y=0,8603 x+8,4933$

$R^{2}=0,8061$

A

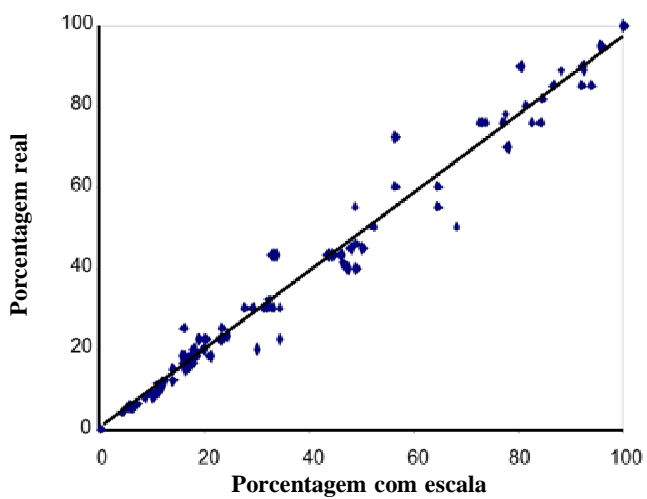

$y=0,9732 x+0,4057$

$R^{2}=0,9784$

B
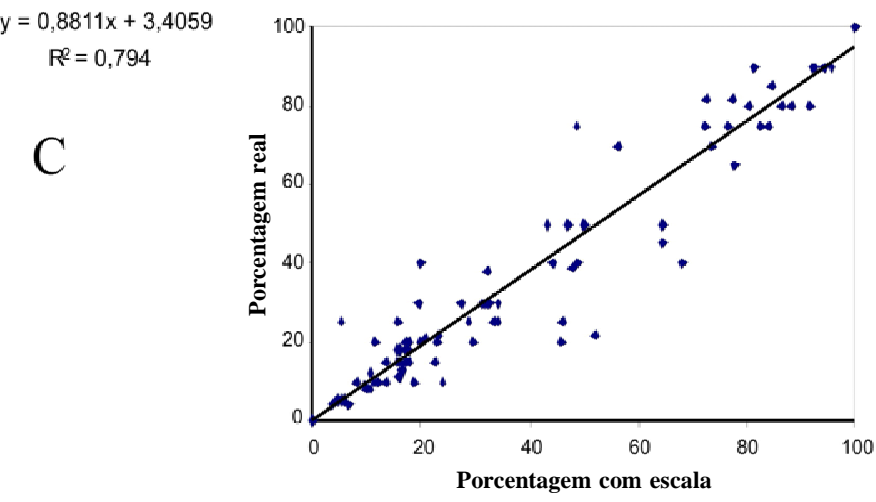

$y=0,951 x+0,2076$ $R^{2}=0,9229$

D

$y=0,935 x-4,0566$

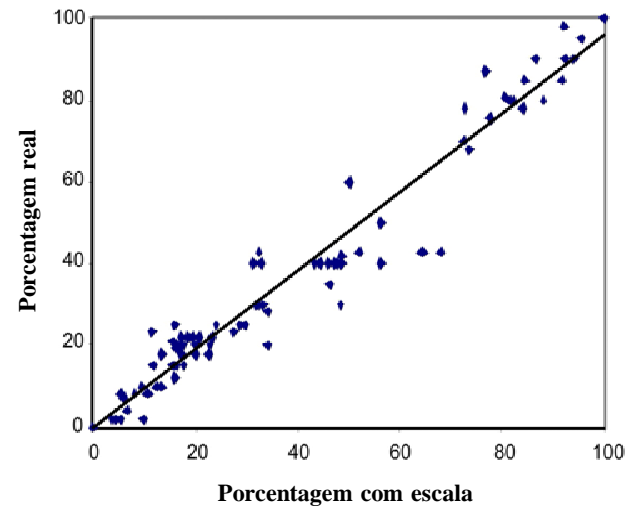

$y=0,9612 x-0,2711$

$R^{2}=0,9563$

F

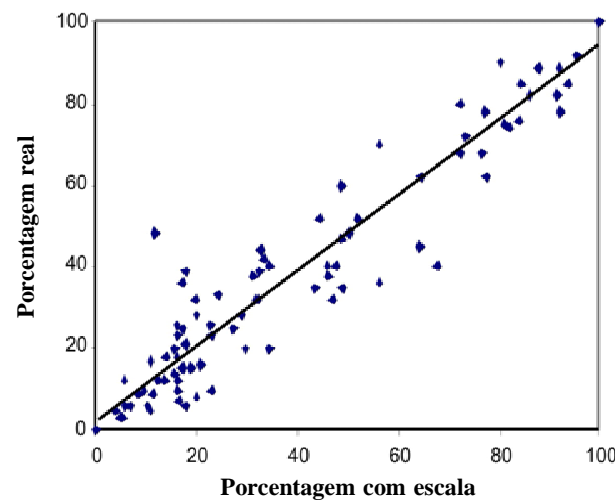

$y=0,9215 x+2,2811$ $R^{2}=0,9155$

$\mathrm{H}$

G

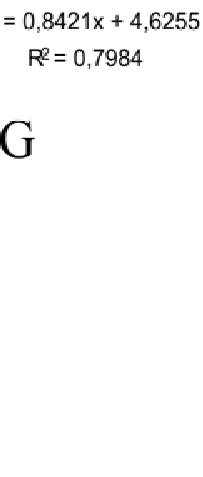

Figura 2. Severidade estimada com o auxílio da escala diagramática elaborada (pontos) e linhas de regressão obtidas entre a severidade real e a estimada (linha cheia) para mofo-cinzento (Amphobotrys ricini) em cachos de mamoneira (Ricinus communis L).A-B avaliador 1 sem e com escala; C-D avaliador 2 sem e com escala; E-F avaliador 3 sem e com escala; G-H avaliador 4 sem e com escala. 


\section{RESULTADOS E DISCUSSÃO}

A incidência e a severidade do mofo-cinzento em mamoneira apresentam valores máximos de $100 \%$, podendo em muitos casos haver queda dos frutos, sendo essas observações já relatadas por Anjani et al. (2), os quais relataram perdas de até $100 \%$ em campos expostos às condições climáticas favoráveis a doença.

A proposta de estabelecer um sistema padronizado para orientar a avaliação da severidade de determinada doença é de grande responsabilidade, pois caso o sistema seja deficiente, o custo de sua utilização pode ser maior do que os benefícios alcançados com seu uso $(12,16)$. Assim, a padronização é altamente desejável, pois a uniformização da metodologia de avaliação de doenças permite comparações entre resultados obtidos em diferentes instituições e localidades (4). A escala diagramática proposta foi composta por seis níveis de severidade: $0 ; 8 ; 22 ; 43 ; 76$ e 100\% (Figura 1). Para seu uso, recomenda-se observar mudanças de cultivares, nutrição, região e demais condições específicas de cada cultivo.

A validação da escala diagramática mostrou resultados bastante promissores quanto ao uso da mesma para a avaliação da doença, estando seus valores de severidade estimados bem próximos aos valores da severidade real (Figura 2). A totalidade dos avaliadores apresentou boa acurácia $\left(\mathrm{R}^{2}=0,94\right)$, sendo os valores de "a" não significativamente diferentes de zero (0) e os valores de "b" não diferentes de um (1) (Tabela 1).

Tabela 1. Estimativas de intercepto $(a)$, do coeficiente angular da reta $(b)$ e do coeficiente de determinação $\left(R^{2}\right)$ de equações de regressão linear simples relacionando estimativas visuais de severidade de cachos de mamoneira provocada por Amphobotrys ricini efetuadas por avaliadores, sem e com auxilio de escala diagramática desenvolvida.

\begin{tabular}{lllllll}
\hline Avaliador & \multicolumn{3}{c}{ Sem escala } & \multicolumn{3}{c}{ Com escala } \\
\hline & $\boldsymbol{a}$ & $\boldsymbol{b}$ & $\boldsymbol{R}^{2}$ & $\boldsymbol{a}$ & $\boldsymbol{b}$ & $\boldsymbol{R}^{2}$ \\
\hline 1 & $8,49^{*}$ & 0,86 & 0,80 & 0,40 & 0,97 & 0,97 \\
2 & $3,41^{*}$ & 0,88 & 0,79 & 0,21 & 0,95 & 0,92 \\
3 & $-4,06^{*}$ & 0,94 & 0,81 & $-0,27$ & 0,96 & 0,95 \\
4 & $4,63^{*}$ & 0,84 & 0,79 & 2,28 & 0,92 & 0,92 \\
Média & 3,12 & 0,88 & 0,80 & 0,66 & 0,95 & 0,94 \\
\hline
\end{tabular}

* Asterísco representa situações onde a hipótese de nulidade $(\mathrm{a}=0$ ou $\mathrm{b}=1)$ foi rejeitada pelo teste $\mathrm{t}(\mathrm{P} \leq 0,01)$.

A precisão é um importante fator a ser considerado na validação de uma escala diagramática, sendo definida como a exatidão de uma operação onde há rigor ou refinamento na medida (4). As estimativas visuais da severidade variaram de 92 a $97 \%\left(\mathrm{R}^{2}\right)$, com média de $94 \%$ entre os avaliadores após a adoção da escala diagramática, enquanto a média sem o uso da escala foi bem inferior $(80 \%)$.

Além das técnicas adotadas para o preparo da escala, Amorim (1) relata que o sucesso do seu uso, medido pela precisão e acurácia na estimativa da severidade da doença, irá depender do treinamento, experiência e da percepção individual de cada avaliador.

A avaliação do comportamento de doenças por meio de metodologia científica e a padronização dos métodos de avaliação de doenças proporcionam maior confiabilidade no monitoramento e auxiliam em futuros estudos sobre a doença.

\section{CONCLUSÃO}

A utilização da escala diagramática com seis níveis para avaliação da severidade do mofo-cinzento da mamoneira a campo mostrou-se precisa e acurada, permitindo a quantificação dos sintomas da doença de maneira precisa. Seu uso em estudos epidemiológicos irá proporcionar informações mais adequadas a respeito do patossistema Amphobotrys ricini - mamoneira.

\section{REFERÊNCIAS BIBLIOGRÁFICAS}

1. Amorim, L. Avaliação de doenças. In: Bergamin Filho A.; Kimati, H; Amorim, L. Manual de fitopatologia. 3. ed. São Paulo: Agronômica Ceres, 1995. v. 1, p. 647-671.

2. Anjani, K.; Raoof, M. A.; Reddy, P. A. V.; Rao, C. H. Sources of resistance to major castor (Ricinus communis L.) diseases. Bulletin de Ressources n. 137, p. 46-48, 2002.

3. Beltrão, N.E.M. et al. Fitologia. In: Azevedo, D.M.P. de Lima, E.F. (Ed.). O agronegócio da mamona no Brasil. Brasília: Embrapa Algodão, 2001. cap. 2, p. 37-61.

4. Bergamin Filho, A.; Amorim, L. Doenças de plantas tropicais: epidemiologia e controle econômico. São Paulo: Agronômica Ceres, 1996. 299p.

5. Campbell, C.L.; Madden, L.V. Introduction to plant disease epidemiology. New York: John Wiley, 1990. 532p.

6. Carvalho, L. O. Mamona (Ricinus communis L.). In: São Paulo (Estado). Secretária de Agricultura e Abastecimento. Coordenadoria de Assistência Técnica Integral. Manual técnico das culturas. 2. ed. Campinas, 1997. cap. 11, p. 349-368.

7. Fornazieri Junior, A. Mamona: uma rica fonte de óleo e de divisas. São Paulo: Ícone, 1986. 72p.

8. Gibelli, F. Projeto poiuretano de óleo de mamona e seus subprodutos. In: Câmara, G. M. S., Chiavegato, E. J. (Coord.). O agronegócio das plantas oleaginosas. Piracicaba: Escola Superior de Agricultura "Luiz de Queiroz"/ Departamento de Produção Vegetal, 2001. cap. 10, p. 181-184.

9. Godfrey, J.T.A. Gray mold of castor bean. Journal of Agricultural Research, Washington, v. 23, n. 9, p. 679-716, 1923.

10. Horsfall, J.C. \& Barrat, R.W. An improved grading system for measuring plant diseases. Phytopathology, 35:665, 1945.

11. Kimati, H. Doenças da mamoneira - Ricinus communis L. In: GALli, F. (coord). Manual de Fitopatologia. São Paulo: Agronômica Ceres, 1980. v. 2, p. 347-351.

12. Leite, R.M.V.B.C.; Amorim, L. Elaboração e validação de escala diagramática para mancha de Alternaria em girassol. Summa Phytopathologica, Botucatu, v. 28, n. 1, p. 14-19, 2002

13. Lima, E.F.; Araújo, A.E.; Batista, F.A.S. Doenças e seu controle. In.: Azevedo, D.M.P. de; Lima E.F. (Ed.). O agronegócio da mamona no Brasil. Brasília: Embrapa Informação Tecnológica, 2001 p. 191-212.

14. Massola Junior, N.S. \& Bedendo, I.P. Doenças da mamoneira. In: Kimati, H. ... [et al.]. Manual de Fitopatologia. 4 ed. São Paulo: Agronômica Ceres, 2005. v. 2: it. p. 445-447.

15. Neergaard, P. Seed pathology. Londres: McMillan, 1979. $839 \mathrm{p}$.

16. Nutter Junior, F.W.; Schultz, P.M. Improving the accuracy and precision of disease assessments: selection of methods and use of computer-aided training programs. Canadian Journal of Plant Pathology, Ottawa, v. 17, n. 1, p. 174-184, 1995 .

17. Savy Filho, A. Melhoramento da mamona. In: Borém, A. Melhoramento de espécies cultivadas. Viçosa: Editora UFV, 1999. p.383-407. 